\title{
THE ALGEBRAIST'S UPPER HALF-PLANE
}

\section{BY DAVID GOSS}

Introduction. The purpose of this article is to introduce the general mathematical community to some recent developments in algebraic geometry and nonarchimedean analysis. Let $r=p^{n}, p$ a rational prime. Then these developments center around the beginnings of an "arithmetic" theory of the polynomial ring $\mathbf{F}_{r}[T]$ over the finite field of $r$ elements. The goal of this theory is to use nonarchimedean analysis to do for $F_{r}[T]$ what classical analysis does for $\mathbf{Z}$. The theory allows us to find direct analogues of many of the classical functions of arithmetic interest in a situation that, at first glance, seems as nonclassical as possible. In the process much will be learned about the polynomials. Much also will be learned about the unique properties of $\mathbf{Z}$ and the classical functions.

One of the exciting aspects of the theory is its great generality. Indeed, we could replace $\mathbf{F}_{r}[T]$ with much more general affine rings of curves over finite fields. More precisely, if $C$ is a projective, smooth curve over $\mathbf{F}_{r}, \infty$ a rational point and $A$ the functions regular away from $\infty$, then we may use $A$ instead of $\mathbf{F}_{r}[T]$. Thus one can, so to speak, get a sense of what analysis might have been forced to if $\mathbf{Z}$ were not a unique factorization domain. Such observations can only come in the present setting since $\mathbf{Q}$ is the only totally real (i.e., all Galois conjugates contained in $\mathbf{R}$ ) field with a unique absolute-value. We have chosen to stick to the polynomials in order to keep the exposition as simple as possible. The jump from the polynomials to more general rings is not terribly large and most essential features appear for $\mathbf{F}_{r}[T]$.

Another exciting aspect is that we begin to see how a given 'arithmetic' situation generates an associated harmonic analysis. As classical harmonic analysis is based on the integers, the one developed here is based on $\mathbf{F}_{r}[T]$. In contrast to classical harmonic analysis which is multiplicative, i.e., based on the exponential function, the one here is based on addition.

Throughout the paper we compare the theory here with the classical one. In this fashion we hope the reader may speedily develop a feel for the subject.

One of the most surprising (and hotly contested) aspects of classical analysis is its harmonic analysis. This centered around the possibility of expanding an arbitrary singly-periodic function in terms of sines and cosines. Since sines and cosines are easily expressed in terms of the exponential function, $e^{(z)}$, the central role of this function is apparent.

Viewed on the complex plane, $e^{(z)}$ has the following very well-known properties: It is never zero, takes addition to multiplication, is invariant under $z \mapsto z+2 \pi i$ and, finally, it is its own derivative. As a consequence, $e^{(z)}$ gives 
rise to an analytic isomorphism of the coset space $\mathbf{C} /(2 \pi i \mathbf{Z})$ with $\mathbf{C}^{*}$.

Two important facts follow immediately. First, if $f$ is a holomorphic function invariant under $z \mapsto z+2 \pi i$, then $f$ descends to a function on $\mathbf{C}^{*}$ and thus automatically has a Fourier-Laurent expansion in $e^{(z)}$. Secondly, the roots of unity are the special values $e^{(a 2 \pi)}, a \in \mathbf{Q}$. Therefore, the simplest and most classical number fields arise naturally out of the study of $e^{(z)}$.

Notice that any rank one $\mathbf{Z}$-submodule of $\mathbf{C}$ (i.e., rank-one "lattice") is of the form $x 2 \pi i \mathbf{Z}, x \in \mathbf{C}^{*}$. So studying $e^{(z)}$ is quite general.

After the singly-periodic holomorphic functions, the next logical object of study is the doubly-periodic functions, i.e., "elliptic functions". These are functions invariant under $z \mapsto z+l$, with $l$ in a "rank-two lattice", i.e., a rank-two, discrete, $\mathbf{Z}$-submodule of $\mathbf{C}$. Here many new phenomena are encountered: As there are no nonconstant holomorphic doubly-periodic functions, one has to study doubly-periodic meromorphic functions. Secondly; there are many "nonisomorphic" (see Chapter 3) rank-two lattices. Thirdly, if $L$ is a rank-two lattice, $\mathbf{C} / L$ (i.e., the associated "elliptic curve", again see Chapter 3) need not have any natural definition over the field of all algebraic numbers, $\overline{\mathbf{Q}}$. (N.B., $\mathbf{C}^{*}$ is the affine line minus the origin and so is defined over $\overline{\mathbf{Q}}$.)

In order to arrive at good "two dimensional" analogues of cyclotomic fields, one does the following: One creates spaces (called "moduli spaces") out of all distinct isomorphism classes of rank two lattices (with, possibly, extra structures). One shows these spaces have the structure of algebraic curves. It is then an important theorem that these curves have very natural definitions over $\overline{\mathbf{Q}}$. (In fact, many important algebraic sets arise over $\overline{\mathbf{Q}}$ in a similar fashion. These spaces are commonly called Shimura-Varieties .)

The key to handling the moduli curves is that they may be described analytically as the upper half-plane $H=\{x+i y \in \mathbf{C} \mid y>0\}$ modulo the action of subgroups of $\mathrm{SL}_{2}(Z)$. Thus functions on $H$ which are holomorphic and invariant under $\mathrm{SL}_{2}(Z)$ have a natural arithmetic interest. However, invariance is too strong a restriction and so one studies functions that are "almost invariant", (see Chapter 4). Such functions are called modular forms.

From definition, modular forms are invariant under $z \mapsto z+n$, some $n \in \mathbf{N}$. As such, in the fashion described above, they have expansions, called " $q$-expansions" in $e^{(2 \pi i z / n)}$. These expansions are the key to the theory and the crucial link between the rank-two and rank-one theories. For instance, the coefficients of the most important modular forms always lie in cyclotomic fields.

Finally, one can only fully appreciate the elegant structure of such expansions by using certain linear operators, called Hecke-Operators. These operators are also important in passing from modular forms to Dirichlet series. With the above tools much arithmetic is developed, e.g., quadratic forms, the $\tau$-function, etc.

Returning now to the polynomials, let $K=\mathrm{F}_{r}((1 / T))$. Our basic fact is that $\mathbf{F}_{r}[T]$ sits discretely in $K$, exactly as $\mathbf{Z}$ sits discretely in $\mathbf{R}$. Further, $K$ is a complete, nonarchimedean, topological field. So we can use its analytic properties to adopt the classical situation to the polynomials.

It is apparent that the classical successes depend totally on the simple fact 
of analytic continuation for complex functions. In standard nonarchimedean analysis analytic continuation is nonexistent. So, first of all, one needs a nonarchimedean theory which has it. Such a theory is called "rigid analytic spaces" and is described in Chapter 1.

Having the power of rigid analysis and with the classical situation in mind, the next goal is to find an adequate notion of a "lattice". One is immediately led to the following definition (due to Drinfeld): A lattice is a discrete, finitely generated, $\mathbf{F}_{r}[T]$-submodule of $\bar{K}$. These lattices give rise to algebraic objects, called "elliptic-modules", exactly as classical lattices give rise to "elliptic curves".

As any lattice is, by definition, finitely generated, it can be described as an increasing union of finite, additive subgroups. This is, of course, a purely finite characteristic occurence and is the basis for the constructions given here.

Associated to rank one lattices, we have an "exponential function". This is an entire, nonarchimedean function which is invariant under translation by the lattice and which is additive. Such functions play the role of the classical exponential function, e.g., certain special values describe finite dimensional, abelian field extensions of $\mathbf{F}_{r}(T)$, etc. However, the reader will note that there is a small degree of ambiguity here and no one function emerges as THE analogue of $e^{(z)}$.

For rank two lattices, we can construct affine curves over $\overline{\mathbf{F}_{r}(T)}$; just as classically we find them over $\overline{\mathbf{Q}}$. It is crucial that we also have a rigid analytic space, $\mathcal{H}$, which plays the role of $H$. We use here $\mathrm{GL}_{2}\left(\mathbf{F}_{r}[T]\right)$ instead of $\mathrm{SL}_{2}(\mathbf{Z})$. Modular forms are then rigid analytic functions with the same invariance as in the classical definition.

As with classical forms, ours have " $q$-expansions". To compute these we develop the additive harmonic analysis. It is based precisely on the finite additive subgroups. These expansions also provide the link with the rank one theory.

With the $q$-expansions and the introduction of Hecke operators for our forms, the classical and finite-characteristic theories diverge sharply. This, no doubt, reflects differences in arithmetic whose full meaning will be understood only over time.

We now summarize the various chapters of this work.

Chapter 1 presents an overview of rigid analytic spaces. These spaces are the global spaces of nonarchimedean analysis; one may, for instance, prove for them analogues of the classical comparison theorems of Serre. The use of this theory will allow us to conclude many results that otherwise would be impossible to prove.

Chapter 2 introduces our basic object of study, the nonarchimedean space $\mathcal{H}$. We summarize the properties of $\mathcal{H}$ that are necessary for modular forms.

In Chapter 3, we restrict our attention to the characteristic- $p$ case of formal Laurent-series fields. We present the basic theorem on algebraization of quotients of $\mathcal{H}$ by discrete subgroups. Our method is to first explain the situation for elliptic modular curves, as this will undoubtedly be more familiar to the reader. Then the same reasoning, with appropriate modifications, carries over to characteristic- $p$.

Chapter 4 introduces modular forms and their $q$-expansions. We describe 
how in the nonarchimedean case one can develop a theory of $q$-expansions. Then we shall show, among other things, the finite dimensionality of spaces of modular forms and the existence of bases of these consisting of forms with algebraic $q$-expansion coefficients.

In Chapter 5 we present the cusp forms and double-cusp forms.

The simplest modular forms are the Eisenstein series, and we discuss them in Chapter 6. The general $q$-expansion computation is then given and one detailed example worked out.

Chapter 7 discusses the Hecke operators for both the elliptic and finite characteristic cases.

Finally Chapter 8 presents some other possible areas of interest.

For detailed mastery of the results, the reader should have a basic knowledge of algebraic curves in all characteristics (as found, for example, in Fulton, Algebraic curves) and a little algebraic number theory, (as found in the beginning of Lang, Algebraic number theory). Although not strictly necessary, some knowledge of the classical theory of elliptic modular forms would be of great help.

The author thanks W. Sinnott and M. Tretkoff for their help in the preparation of this work.

\section{Table of Contents}

Chapter 1. Rigid analytic spaces

(a) Standard nonarchimedean analysis

(b) Tate's affine theory

(c) Globalization

Chapter 2. The algebraist's upper half-plane

Chapter 3. The classical theory and the theory over a formal Laurent-series field

(a) Summary of the classical situation

(b) The Laurent-series case

Chapter 4. Modular forms and $q$-expansions

(a) The classical case

(b) The Laurent-series case

Chapter 5. Cusp forms and double-cusp forms

Chapter 6. Eisenstein series and additive harmonic analysis

(a) Classical Eisenstein series

(b) Characteristic- $p$ Eisenstein series

(c) Additive harmonic analysis

(d) $q$-expansion in finite characteristic

Chapter 7. Hecke operators

(a) Hecke operators for congruence subgroups of $\mathrm{SL}_{2}(\mathrm{Z})$

(b) Hecke operators in the Laurent-series case

Chapter 8. Complements

Bibliography

1. Rigid analytic spaces. The theory of rigid spaces is the global theory of nonarchimedean analysis. It is a very useful device whose purpose is to allow one to perform classical analysis. We shall give the main ideas of the 
construction so that the reader may have confidence in later developments. The construction has three parts.

(a) Standard nonarchimedean analysis. Let $K$ be a discretely-valued local field. Thus, $K$ can be either a finite extension of $Q_{p}$ (the field of $p$-adic numbers) or a formal Laurent-series field over a finite field. Let ||$: K \rightarrow \mathbf{R}$ be the valuation on $K$. Then | | satisfies

$$
|x \cdot y|=|x| \cdot|y|
$$

and

$$
|x+y| \leqslant \operatorname{Max}\{|x|,|y|\} .
$$

Further, $K$ is complete with respect to the induced metric. Let $\bar{K}$ be the algebraic closure of $K$. It is well known that | | extends uniquely to $\bar{K}$, (see [8], for instance). However, $\bar{K}$ is neither complete nor discretely-valued. We let $(\bar{K})^{c}$ be its completion under || . It is a theorem that $(\bar{K})^{c}$ is still algebraically closed. It is analogous to $\mathbf{C}$ in that it is both complete and algebraically closed. It is, however, not locally compact.

The set $\theta=\{x \in K|| x \mid \leqslant 1\}$ is compact. From the second property of || we see it is a ring. It is called the ring of integers of $K$. The set $M=\{x \in \vartheta \mid$ $|x|<1\}$ is thus an ideal. As || is discrete, $M$ and $\theta$ are both open and closed. Thus, $\theta / M$ is finite. As $M$ is maximal, $\theta / M$ is a field.

Now let $\sum_{n=0}^{\infty} a_{n}$ be an infinite series with $\left\{a_{n}\right\} \subseteq K$. As classically true, for there to be convergence $a_{n}$ must tend to zero as $n \rightarrow \infty$. One of the big advantages of analysis over $K$ (or any complete nonarchimedean field) is that this condition is also sufficient. Indeed let $T_{n}=\operatorname{Max}_{i \geqslant n+1}\left\{\left|a_{i}\right|\right\}$ and let $S_{j}=\sum_{i=0}^{j} a_{i}$. Then, for $m>n,\left|S_{m}-S_{n}\right| \leqslant T_{n}$ by the nonarchimedean property of || . Thus, $\left\{S_{j}\right\}$ is Cauchy and so converges.

Now let $f(x)=\sum a_{i} x^{i}$ be a power series with coefficients in $K$. Let $z \in K$. Then a necessary and sufficient condition that $f$ converge at $z$ is that $a_{i} z^{i} \rightarrow 0$ as $i \rightarrow \infty$. Using this notion we see that many of the classical theorems on power series are valid. For instance, if $f$ converges at $z$ then it converges for all $y \in K$ with $|y| \leqslant|z|$, etc.

Now suppose $f$ is entire; i.e. $f$ converges for all $z \in K$. Then, we have a very strong form of the Weierstrass Product Formula. We state it below; a proof may be found in [10]. For now, we consider $f$ as a function on $(\bar{K})^{c}$ in the natural fashion.

THEOREM 1.1. Let $f(x)=\sum_{i \geqslant 0}^{\infty} a_{i} x^{i}$ be an entire function. Then, the zeroes of f belong to $\bar{K}$. Further,

$$
f(x)=c x^{k} \prod_{0 \neq \alpha}(1-x / \alpha)
$$

where $\alpha$ ranges over the nonzero roots (with multiplicity).

COROllary 1.2. An entire function is determined up to a constant by its divisor.

Conversely, once we take into account the Galois action, it is easy to see how to construct entire functions by using products of the above type. 
Notice that Corollary 1.2 provides an intrinsic explanation for the wellknown fact that, over $Q_{p}$, the usual exponential function has a finite radius of convergence. A bit later we will construct nonarchimedean entire functions that play a role similar to $e^{(x)}$. As a further introduction to standard non-archimedean analysis, the reader may consult the book of N. Koblitz, number 58 in the Graduate Texts in Mathematics series.

The basic difficulty with this form of analysis is that there does not exist a straightforward notion of analytic continuation. In fact, let $\mathcal{O}^{*}=\{x \in \mathcal{O} \mid$ $|x|=1$. Then $\theta^{*}$ is also compact-open and $\theta=\theta^{*} \cup M$. Now let $f$ : $\vartheta \rightarrow K$ be given by $f\left(\theta^{*}\right)=1$ and $f(M)=0$. Obviously, $f$ can be expanded locally in a power-series; a horrible state of affairs!

(b) Tate's affine theory [13]. The idea of this step is beautifully simple; if there were analytic continuation, the "global" functions on $\theta$ would be those power series with radius of convergence $\geqslant 1$. These series form a ring, and we make rings of this form our first objects of study.

Definition 1.3. Let $D^{n}=\left\{\left(x_{1}, \ldots, x_{n}\right) \in K^{n}|| x_{i} \mid \leqslant 1\right.$ for all $\left.i\right\} . D^{n}$ is called the $n$th polydisc.

Note: In this notation, $D^{1}=0$ as a point set.

Definition 1.4. Let $K\left\{x_{1}, \ldots, x_{n}\right\}$ be the ring of all formal power-series which converge on $D^{n}$.

We know, from above, that a series is in $K\left\{x_{1}, \ldots, x_{n}\right\}$ iff its coefficients tend to 0 as the degree of the monomial tends to $\infty$.

Since $K$ is discretely-valued, it is not hard to see that $K\left\{x_{1}, \ldots, x_{n}\right\}$ is noetherian. Let $J \subseteq K\left\{x_{1}, \ldots, x_{n}\right\}$ be an ideal. Any ring isomorphic to $K\left\{x_{1}, \ldots, x_{n}\right\} / J$, for some $J$, is called a Tate algebra.

Tate's main result about these algebras is that every maximal ideal is of finite codimension as a vector space over $K$. In the case of $K\left\{x_{1}, \ldots, x_{n}\right\}$ this can be interpreted in a geometric (and more classical) fashion as follows. Let $L$ be a finite extension of $K$. We know that || has a unique extension to $L$. Let $\left(a_{1}, \ldots, a_{n}\right) \in L^{n}$ with $\left|a_{i}\right| \leqslant 1$ for all $i$. Then we have a homomorphism $K\left\{x_{1}, \ldots, x_{n}\right\} \rightarrow L$ given by $x_{i} \rightarrow a_{i}$ all $i$. The kernel is a maximal ideal. Tate's theorem then says every maximal ideal arises in this fashion. Of course, if $\sigma$ is an automorphism of $\bar{K}$ over $K$, the elements $\left(a_{1}, \ldots, a_{n}\right)$ and $\left(\sigma\left(a_{1}\right), \ldots, \sigma\left(a_{n}\right)\right)$ give rise to the same ideal. Thus, we see that the ring $K\left\{x_{1}, \ldots, x_{n}\right\}$ "represents" the polydisc via its maximal ideals; these correspond to the quotient of the polydisc over $\bar{K}\left(=\left\{\left(x_{1}, \ldots, x_{n}\right) \in(\bar{K})^{n}|| x_{i} \mid \leqslant\right.\right.$ 1 ; all $i\})$ by the Galois action. For any Tate algebra $B$ we let $\operatorname{Max}(B)$ be the set of its maximal ideals. We now see that $\operatorname{Max}(B)$ is, in general, some analytic subset of a polydisc; i.e. is given by analytic equations.

From this approach one can prove corollaries in analysis. We cite two, and we refer the reader to Tate's article for the proofs. The first is the following: Let $B$ be a Tate algebra containing no nilpotent functions (i.e. $x^{n}=0$ iff $x=0 ; B$ is said to be "reduced") and let $\left\{f_{n}\right\}$ be a sequence in $B$. Suppose that on $\operatorname{Max}(B)$ this sequence is uniformly convergent. Let $f(z)=\lim \left\{f_{n}(z)\right\}$ for $z \in \operatorname{Max}(B)$. Then, the first fact is that $f \in B$; i.e. uniform limits of analytic functions are analytic. The second fact is that an element $f \in B$ which is never zero on "geometric points" has a reciprocal in $B$. (A geometric point is a point in the analytic subset of the polydisc over $\bar{K}$ that is given by 
the zeroes of the equations defining $B$.) In other words, a function which has no zeroes in any extension of $K$ has a holomorphic reciprocal.

Taking his cue from algebraic geometry, Tate makes the spaces of maximal ideals his basic "affine" objects of study. Let $B_{1}, B_{2}$ be two Tate algebras and $\phi: B_{1} \rightarrow B_{2}$ an algebra map. Then we get an associated map $\phi^{*}: \operatorname{Max}\left(B_{2}\right) \rightarrow$ $\operatorname{Max}\left(B_{1}\right)$ by taking the inverse image of maximal ideals. We thus define the morphisms of affine rigid spaces as being dual to algebra maps of the associated Tate algebra. We call the Tate algebra the algebra of rigid functions.

Affine subspaces of affine spaces are defined by "representation." More precisely, let $Y$ be subset of $X=\operatorname{Max}(B)$. Suppose we have another Tate algebra $B_{1}$, with a map $\phi^{*}: \operatorname{Max}\left(B_{1}\right) \rightarrow X$ (dual to $\phi: B \rightarrow B_{1}$ ) such that: (1) $\phi^{*}\left(\operatorname{Max}\left(B_{1}\right)\right) \subseteq Y$, (2) If $B_{2}$ is a third Tate algebra having a map $\phi_{1}^{*}$ : $\operatorname{Max}\left(B_{2}\right) \rightarrow X$ with image in $Y$, then there exists a unique map $\phi_{2}: B_{1} \rightarrow B_{2}$ so that the following triangle is commutative.

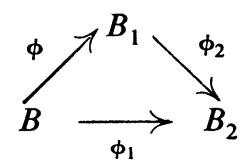

If such a $B_{1}$ exists we say that $Y$ is an affine subspace of $\operatorname{Max}(B)$ and denote $B_{1}$ by $B_{Y}$. Notice that by the universal property $\operatorname{Max}\left(B_{Y}\right) \simeq Y$ as point sets.

For example let $B=K\left\{X_{1}, \ldots, X_{n}\right\} /\left(f_{1}, \ldots, f_{m}\right), f \in B$ and $c \in K$. Then the place where $|f(x)| \leqslant|c|$ in $\operatorname{Max}(B)$ is represented by $K\left\{X_{1}, \ldots, X_{n}, y\right\} /\left(f_{1}, \ldots, f_{m}, f-y c\right)$.

If $Y_{0}, Y_{1}$ are two affine subspaces of $\operatorname{Max}(B)$, then $Y_{0} \cap Y$ is also. The associated algebra is the "completed" tensor product, $B_{Y_{0}} \hat{\otimes} B_{Y_{1}}$, i.e., we take the tensor product and then include all uniformly convergent limits.

(c) Globalization ([6], [7]). The final step is to put the affines together in such a way as to force analytic continuation. The idea here comes from the following theorem of Grauert and Gerritzen (see [5]).

THEOREM 1.5. Let $U=\operatorname{Max}(B)$, be an affine rigid space. Let $\left\{U_{i}\right\}$ be a covering of $U$ by a finite number of analytic affine open subsets. Then $\left\{U_{i}\right\}$ has an acyclic $\check{C}$ ech complex.

In down to earth terms, the theorem says we may define functions locally; i.e., if $\left\{f_{i}\right\}$ is a set of rigid functions on the $U_{i}$ which agree on overlaps, then they give rise to a rigid function on $U$. The example in (a) is ruled out because the set $\{x \in \bar{K}|| x \mid<1\}$ can not be covered by a finite number of affines.

Finally, then, a rigid analytic space is a space $X$ together with a given covering $\left\{U_{i}\right\}$ of $X$ by affines and the following axioms on the use of this covering:

(1) Let $U \subseteq X$. We say that $f$ is a rigid function on $U$ iff for all $V$ affine, $V$ an affine subspace of $U_{i}$ some $i$ and $V \subseteq U$, we have $f$ is rigid on $V$.

(2) Let $U \subseteq X$ : We say $U$ is admissible iff there is a covering $\left\{V_{j}^{i}\right\}$ of $U \cap U_{i}$, all $i$, of affine subspaces of $U_{i}$ so that if $V \subseteq U \cap U_{i}$ is the morphic image of any affine then $V$ is contained in a finite number of the $\left\{V_{j}^{i}\right\}$. (These spaces are the correct ones for patching.) 
(3) Let $Y, U$ be two admissible subsets of $X$. Then we say that $\{Y, U\}$ is an admissible cover of $U \cup Y$ (and so we can patch functions) iff for every affine subspace $V \subseteq U_{i}$, some $i$, with $V \subseteq U \cup Y$, the covering of $V$, obtained by the coverings of $V \cap U$ and $V \cap Y$, may be refined to a finite affine cover. The general notion of an admissible covering by an arbitrary number of admissibles is similar.

One can only patch functions on admissible subsets in the fashion given by (3), or else we should have far too many functions. Our definitions are the minimum that we could have in accordance with Theorem 1.5. The reader should note that with our axioms rigid spaces are examples of spaces with a "Grothendieck Topology".

Morphisms of rigid spaces are mappings pulling rigid functions back to rigid functions.

For instance, $A_{K}^{n}$ may be given a rigid structure by taking as our covering discs of increasingly large radii. Further, any projective variety has a rigid structure involving only finitely many affines. On projective varieties the G.A.G.A. theorems of Serre hold (see Kiehl [6]) e.g., a meromorphic function is an algebraic function (of course the reverse is also true).

Let $X$ be a rigid analytic space defined over $K$. Suppose that $X$ is reduced in that it has no nilpotent functions. We say that $X$ is connected iff a rigid function with zero Taylor series at a point is everywhere zero. We say $X$ is geometrically connected iff it is connected when viewed as an analyc space over all finite extensions $L$ of $K$.

The moral of the construction is this: We have seen the main problem is the existence of too many open sets. So, we reduce the number of "admissible" open sets until we force analytic continuation. Then, as often happens with modern algebra, it's "the good old days" again.

2. The algebraist's upper half-plane. We now introduce our basic rigid analytic space. Recall that the classical upper and lower half-planes can be described as $\{x \in \mathbf{C} \mid x \notin \mathbf{R}\}$. This definition also makes sense for local nonarchimedean fields $\bar{K}$ !

Definition 2.1. We set $\mathcal{H}=\{x \in \bar{K} \mid x \notin K\}$. $\mathcal{H}$ was originally introduced by V. G. Drinfeld in [1], (and denoted $\Omega^{2}$ ).

As a point set, $\mathcal{K}$ is clearly open in $\bar{K} ; \bar{K}$ being equipped with the topology induced by || . Further, $\mathcal{H}$ has an action of $\mathrm{GL}_{2}(K)$ on it: Let $g=\left(\begin{array}{ll}a & c \\ b & d\end{array}\right) \in$ $\mathrm{GL}_{2}(K)$. Then $(g, z) \mapsto(a z+b) /(c z+d)$.

Drinfeld shows the following (see [1]).

THEOREM 2.2. (a) $\mathcal{H}$ has a rigid analytic structure on it as an admissible open in $\mathbf{A}^{\mathbf{l}}$. This structure is defined over $K$.

(b) The action of $G L_{2}(K)$ extends to rigid analytic isomorphisms of $\mathcal{H}$.

(c) $\mathcal{H}$ is a geometrically connected rigid analytic space.

(d) Let $\Gamma \subseteq G L_{2}(K)$ be a discrete group. Then the quotient space $\Gamma \backslash \mathcal{H}$, as a point set, may be given a natural rigid analytic structure. The natural map $\mathcal{H} \rightarrow \Gamma \backslash \mathcal{H}$ is a mapping of rigid spaces.

The quotient in (d) is constructed by finding an admissible affine covering of $\mathcal{H}$, which is permuted around by $\Gamma$ and which has finite isotropy at each 
affine. One is thus immediately reduced to the simple task of finding quotients of affine spaces (i.e., Tate algebras) by finite groups.

As $\mathcal{H}$ is a rigid open subset of $\mathbf{A}^{1}$, it is smooth. Thus $\Gamma \backslash \mathcal{H}$ is normal and, being cne-dimensional, also smooth.

Definition 2.3. For $x \in \mathcal{H}$, let $d(x)=\operatorname{Min}_{a \in K}\{|x-a|\}$. It is clear that for $x \in \mathcal{H}, d(x) \neq 0$. Further, the places where $d(x)$ is constant are seen easily to be open. In [2], the following is shown.

Proposition 2.4. (a) Let $c \in \mathbf{R}$. Then the subspace of $\mathcal{H}$ given by $\{x \in \mathcal{H} \mid d(x) \geqslant c\}$ is a rigid analytic open subset of $\mathcal{H}$.

(b) Let $i \in \mathrm{N}^{+}$and $U_{i}=\{x \in \mathcal{H} \mid d(x) \geqslant 1 / i\}$. Then, to show a sequence of rigid functions on $\mathcal{H}$ converges to a rigid function, it is sufficient to show it for $U_{i}$, all $i$.

EXAMPLE. Let $c \in K$, with $|c|<1$. To give a feel for the rigid structure on $\mathcal{H}$, we show how to represent the space $V=\{x \in \mathcal{H}|| x \mid \leqslant 1$ and $d(x) \geqslant|c|\}$. First, note that $D^{1} \cap K=\mathcal{O}$ is compact. Let $\left\{b_{1}, \ldots, b_{n}\right\}$ be a collection of elements in $\mathcal{O}$ so that for any $x \in \mathcal{O}$, there exists $i$ with $\left|x-b_{i}\right|<|c|$. Then $V$ is the affine open given by $\left|z-b_{i}\right| \geqslant|c|$, all $i$. Indeed, $V$ is clearly contained in this space. Now, let $x$ be in this space. Suppose $d(x)<|c|$. Let $a \in K$ with $|x-a|<|c|$. Clearly $|a|<1$. Further, for some $i,\left|b_{i}-a\right|<|c|$. Therefore, by the nonarchimedean property,

$$
\left|x-b_{i}\right|=\left|x-a+a-b_{i}\right|=\operatorname{Max}\left\{|x-a|,\left|a-b_{i}\right|\right\}<|c| .
$$

This is a contradiction.

3. The classical theory and the theory over a formal Laurent-series field.

(a) Summary of the classical situation. Although $\mathcal{H}$ is defined for any nonarchimedean local field, it is especially interesting in the case where $K$ is a formal Laurent-series field. The reason for this lies in the existence of many interesting discrete subgroups of $\mathrm{GL}_{2}(K)$. Indeed, let $C$ be a smooth, projective curve, $k$ the function field and $\infty$ a point with $k_{\infty}$ (i.e. the completion of $k$ at $\infty$ ) isomorphic to $K$. Further, let $A$ be the Dedekind ring of functions on $C$ holomorphic away from $\infty$. Then, $\mathrm{GL}_{2}(A) \subseteq \mathrm{GL}_{2}(K)$ is a discrete subgroup.

For simplicity of exposition, we now let $k=\mathbf{F}_{r}(T), A=\mathbf{F}_{r}[T]$ and $\infty$ the point at infinity on $\mathbf{P}^{1}$. From the division algorithm we see $\mathbf{F}_{r}[T]$ is a p.i.d.; so all torsion free finitely generated modules are free. For $i \in \mathbf{F}_{r}[T]$ we let $D(i)$ be its degree.

We see the situation parallels the classical situation: $\mathbf{F}_{r}[T]$ plays the role of $\mathbf{Z}, \mathbf{F}_{r}(T)$ the role of $Q, k_{\infty}=K$ the role of $\mathbf{R}$ and $\mathrm{GL}_{2}(A)$ the role of $\mathrm{SL}_{2}(\mathbf{Z})$ (acting on $H$, the classical upper half-plane, in the usual fashion, $\left(\left(\begin{array}{ll}a & c \\ b & d\end{array}\right), z\right) \rightarrow$ $(a z+b) /(c z+d))$.

Definition 3.1. (a) In the classical situation, let $\Gamma=\mathrm{SL}_{2}(\mathbf{Z})$. If $J \subseteq Z$ is a nonzero ideal we set $\Gamma(J)=\operatorname{Ker}\left\{\Gamma \rightarrow \mathrm{SL}_{2}(\mathbf{Z} / J)\right\}$.

(b) In the nonarchimedean situation, let $\Gamma=\mathrm{GL}_{2}(A)$. If $J \subseteq A$ is a nonzero ideal we let $\Gamma(J)=\operatorname{Ker}\left\{\Gamma \rightarrow \mathrm{GL}_{2}(A / J)\right\}$. (Notice $\Gamma((1))=\Gamma$ is both cases.)

Such subgroups are called principal congruence subgroups. 
In the case of the principal congruence subgroups of $\mathrm{SL}_{2}(\mathbf{Z})$, an enormous amount of information is known, (see [12]). We summarize some of this in the following theorem.

THEOREM 3.2. Let $G$ be a principal congruence subgroup of $S L_{2}(\mathbf{Z})$. Let $X_{G}=G \backslash H$. Then (a) $X_{G}$ is a smooth Riemann surface. It may be compactified to a smooth compact Riemann surface by adding a finite number of points (called cusps). These points are in one to one correspondence with $G \backslash \mathbf{P}^{1}(Q)$ (e.g. for $S L_{2}(\mathbf{Z})$ there is one cusp, etc).

(b) $X_{G}$ may be given a natural structure as an affine algebraic curve defined over some cyclotomic field.

The idea behind the proof of (b) lies with the interpretation of $X_{G}$ as parametrizing isomorphism classes of "elliptic curves". More precisely, let $\tau \in H$. Then, the $\mathbf{Z}$-submodule of $\mathbf{C}, L_{\tau}=\mathbf{Z}+\mathbf{Z} \tau$ is a rank two "lattice"; i.e. it is discrete and of rank two as $\mathbf{Z}$-module. It is easy to see that a general rank two lattice may be written $r(\mathbf{Z}+\mathbf{Z} \tau)$ with $r \in \mathbf{C}^{*}$. We shall see in a moment that the lattices $\mathbf{Z}+\mathbf{Z} \tau$ and $r(\mathbf{Z}+\mathbf{Z} \tau)$ define isomorphic objects. Thus, we will be able to confine ourselves to $L_{\tau}, \tau \in H$. The map $L_{\tau} \stackrel{r}{\rightarrow} r L_{\tau}$ is called a homothety. Now $\mathbf{C} / L_{\tau}$ is a compact Riemann surface that may be given the structure of a nonsingular cubic in $\mathbf{P}^{2}(\mathbf{C})$. The induced $\mathbf{Z}$-module structure on $\mathrm{C} / L_{\tau}$ may be transferred to the cubic. Further, on the cubic the structure is given by polynomial equations. In other words, the analytic space $\mathbf{C} / L_{\tau}$ may be given a purely algebraic interpretation as a cubic with addition. Such cubics are called elliptic curves and it is a theorem that, over $\mathbf{C}$, all such cubics arise from rank two lattices. Now let $r \in \mathbf{C}^{*}$. Then $r$ gives rise to an isomorphism $\mathrm{C} / L_{\tau} \stackrel{r}{\rightarrow} \mathrm{C} / r L_{\tau}$. The same is true for the corresponding cubics and any isomorphism arises this way. Therefore, since the underlying isomorphism class of the elliptic curve depends neither on a basis for the lattice, nor its homothety class, we see that $\mathrm{SL}_{2}(\mathrm{Z}) \backslash H$ parametrizes isomorphism classes of elliptic curves over $\mathbf{C}$.

Suppose $n \in \mathbf{N}$ and let $E$ be an elliptic curve over $\mathbf{C}$. Further, let $E[n]=$ $\{x \in E \mid n \cdot x=0\}$. From the description of $E$ as $\mathbf{C} / L_{\tau}$, for some $\tau \in H$, we see $E[n] \stackrel{\sim}{\rightarrow} \mathbf{Z} /(n) \oplus \mathbf{Z} /(n)$. A level $(n)$-structure is a choice of basis for this free $\mathbf{Z} /(n)$-module. One sees as above that, over $\mathbf{C}, \Gamma((n)) \backslash H$ parametrizes elliptic curves with level $(n)$-structure.

Now the concepts of elliptic curve and level structure make sense over $Q$. As these concepts are algebraic, all isomorphisms can be made over finite extensions of $Q$. Therefore, the relations characterizing isomorphism classes of elliptic curves with level (n)-structure are definable over finite extensions of $Q$, (in fact, cyclotomic fields). Since these relations are precisely the underlying equations of $X_{\Gamma((n))}$ we have succeeded (modulo rigorous proof!) of giving $X_{\Gamma((n))}$ the desired algebraic structure over $\bar{Q}$.

If we use the same arguments for rank one lattices $\subset \mathbf{C}$, we would be describing cyclotomic fields. Indeed, the usual exponential function $e^{(z)}$ gives an isomorphism $\mathbf{C} /(2 \pi i \cdot \mathbf{Z}) \stackrel{\sim}{\rightarrow} \mathbf{C}^{*}$, and the division points are precisely roots of unity.

(b) The Laurent-series case. We now return to the characteristic-p case. 
Here, we want to follow exactly the same program as for $\operatorname{SL}_{2}(\mathbf{Z})$, so it is necessary to create the analogues of elliptic curves, etc.

It will be convenient to handle the ranks one and two cases together. We shall be rather brief; for more information in the very simple case of $\mathbf{F}_{r}[T]$ the reader can consult [4]. (Note, in [4], only the rank one case is discussed. However, the general case is exactly the same.)

Our first definition is a direct carry over of the classical one. Recall, that $\overline{k_{\infty}}$ has the topology induced from || .

Definition 3.3. An $A$-lattice $M$ is a finitely-generated, discrete $A$-submodule of $\overline{k_{\infty}}$. Its rank is the rank of the underlying free module.

By discrete we mean that the intersection of $M$ with every ball around the origin is finite. One can see that every rank one lattice is of the form $A i$ with $i \in \bar{k}_{\infty}^{*}$. Any rank two lattice is of the form $i(A+A \tau)$ with $i \in \bar{k}_{\infty}^{*}$ and $\tau \in \mathcal{H}$. (As with $\mathbf{Z}$, we shall eventually only need to confine ourselves to lattices of the form $L_{\tau}=A+A \tau, \tau \in \mathcal{H}$.)

Now let $L$ be a rank one or two lattice. As a group we can form $\overline{k_{\infty}} / L$. The fundamental fact is, like over $\mathbf{C}, \overline{k_{\infty}} / L$ has an algebraic structure. The construction goes as follows: Form the function

$$
e_{L}(z)=z \prod_{0 \neq \alpha \in L}(1-z / \alpha) .
$$

Because $L$ is discrete, the results in Chapter $1, \S(\mathrm{a})$, tell us $e_{L}$ is entire as a function defined over some finite extension of $k_{\infty}$. It's most basic property is that it is additive. Indeed, we can write $L=\cup L_{i}$, where $L_{i}$ is a finite $\mathbf{F}_{r}$ vector space. Thus, $e_{L}(z)=\lim e_{L_{i}}(z)$ where $e_{L_{i}}$ is formed in the obvious fashion. By definition, $e_{L_{i}}(z)$ is a polynomial whose roots form an additive group. It is classical that $e_{L_{i}}(z)$ is additive; $e_{L_{i}}(z+y)=e_{L_{i}}(z)+e_{L_{i}}(y)$. Thus, $e_{L}(z)$ is also additive and even $F_{r}$-linear. Its derivative, $e_{L}^{\prime}(z)$, is identically 1 .

Thus, $e_{L}$ gives an isomorphism of groups, $k_{\infty} / L \stackrel{\sim}{\rightarrow} k_{\infty}$. So, as we did for $\mathbf{Z}$ and complex lattices, we can use $e_{L}$ to carry over the $A$-module structure on $\overline{k_{\infty}} / L$ to $\overline{k_{\infty}}$. We shall denote this by $(a, z) \mapsto a * z ; a \in A, z \in \overline{k_{\infty}}$. By definition, $e_{L}(a z)=a * e_{L}(z)$. But $e_{L}(a z)$ and

$$
a e_{L}(z) \prod_{\substack{\alpha \in a^{-1} L / L \\ 0 \neq \alpha}}\left(1-e_{L}(z) / e_{L}(\alpha)\right)
$$

have the same divisors and derivative. So, by Corollary 1.2, they are equal. Therefore, we have the basic formula,

$$
a * y=a y \prod_{\substack{\alpha \in a^{-1} L / L \\ 0 \neq \alpha}}\left(1-y / e_{L}(\alpha)\right) .
$$

In particular, $\zeta * y=\zeta y$ for $\zeta \in \mathbf{F}_{r}$.

Now let $F$ be the $r$ th power map; i.e. $F(z)=z^{r}$. Let $\bar{k}_{\infty}\{F\}$ be the polynomials in $F ; \sum a_{i} F^{i}$. Under composition, these polynomials form a ring. This ring is noncommutative since $(a F)(b F)=a b^{2} F^{2}$ etc. Nevertheless, via $e_{L}$, we have constructed a homomorphism $\phi: a \rightarrow \phi(a)=a *()$, from $A$ to $\bar{k}_{\infty}\{F\}$. (So, to compute $\phi\left(T^{2}\right)$ we compose $\phi(T)$ with itself, etc.) This 
homomorphism is even $\mathbf{F}_{r}$-linear. By counting the number of elements in $a^{-1} L / L \stackrel{\sim}{\rightarrow}(A /(a))^{\text {rank } L}$, and setting $d=\operatorname{rank} L$, we see we can write

$$
\phi(a)=a *()=a F^{0}+\sum_{i=0}^{d D(a)} a_{i} F^{i} ; \quad a_{i} \in \bar{k}_{\infty}, a_{d D(a)} \neq 0 .
$$

Finally, as $\phi$ is $\mathbf{F}_{r}$-linear, it is determined by

$$
\phi(T)=T F^{0}+\sum_{i=0}^{d} a_{i} F^{i} .
$$

If $d=1$,

$$
\phi(T)=T F^{0}+a F ; \quad a \in \bar{k}_{\infty}^{*} .
$$

If $d=2$,

$$
\phi(T)=T F^{0}+a_{1} F+a_{2} F^{2} ; \quad a_{1} \in \bar{k}_{\infty}, a_{2} \in \bar{k}_{\infty}^{*} .
$$

The rank of $\phi$ is defined to be the rank of the corresponding lattice.

The wonderful fact, of course, is that $\phi$ is a purely algebraic object. It makes sense over any field containing $k$. Via it, $\bar{k}_{\infty}$ acquires a new $A$-module structure, $(a, z) \mapsto \phi(a)(z)$. Such modules are called elliptic or Drinfeld modules. As in the elliptic curve case, one can prove that all such modules arise from lattices. Further, homothetic lattices give rise to isomorphic modules, etc.

EXAMPLE 3.4. We shall present here the details concerning a particular rank one elliptic module $C$, given by $C(T)=T F^{0}-F$. This module was studied by Carlitz in the 1930s; see [4]. First of all, we want to describe $e_{L}$, where $L$ is the lattice corresponding to $C$. For $i \in N$, let $[i]=T^{r^{i}}-T$. Further, let $D_{0}=1$ and $D_{i}=[1]^{i} \cdots[i]$ for $i>0$. From the fact that $e_{L}$ is $\mathbf{F}_{r}$-linear we see its derivative, $e_{L}^{\prime}(z)$, is identically 1 . Thus, $e_{L}(z)=z+\sum_{i=1}^{\infty} c_{i} z^{r^{\prime}}$. From the equation

$$
e_{L}(T z)=T\left(e_{L}(z)\right)-e_{L}(z)^{r},
$$

we find, by induction,

$$
e_{L}(z)=\sum_{h=0}^{\infty}(-1)^{h} \frac{z^{r^{h}}}{D_{h}}
$$

Now, let $\lambda=\prod_{i=1}^{\infty}(1-[i] /[i+1])$. Note that $\lambda$ is a unit at $\infty$, i.e. $|\lambda|=1$. Let $\alpha$ be any $(r-1)$ st root of [1]. We set $\bar{\lambda}=\alpha \lambda$. One can show $L=\mathbf{F}_{r}[T] \bar{\lambda}$ and that $\bar{\lambda}$ is transcendental. (In [4], $\bar{\lambda}$ is denoted by $\bar{\pi}$.) Notice that $L$ is invariant of the choice of $\alpha$.

From our discussion, we see $e_{L}$ is very similar to the classical exponential function. Also $\bar{\lambda}$ is strikingly similar to $2 \pi i$.

We could just have easily worked with the modules $C_{\zeta}, \zeta \in \mathbf{F}_{r}^{*}$, given by $C_{\zeta}(T)=T F^{0}-\zeta F$. We leave the easy modifications to the reader.

Let $a \in A$ and let $E=(\phi), \phi: A \rightarrow \bar{k}_{\infty}\{F\}$, be an elliptic module of rank $d$. Let $E[(a)]=\operatorname{ker}\left(\phi(a): \bar{k}_{\infty} \rightarrow \vec{k}_{\infty}\right) . E[(a)]$ is a natural $A$-module. From the analytic description, $E[(a)] \simeq A /(a) \oplus \cdots \oplus A /(a)$, where $A /(a)$ appears $m$ times, $m$ being the rank of $\phi$. A level $(a)$-structure is a choice of basis for this free $A /(a)$-module. 
Now, with the above algebraic objects, we can simply repeat the discussion given for principal congruence subgroups of $\mathrm{SL}_{2}(\mathbf{Z})$ to conclude the following theorem in the case of rank-two lattices.

THEOREM 3.5 (see [1]). Let $G$ be a principal subgroup of $G L_{2}(A)$.

(1) $G \backslash \mathcal{H}=X_{G}$ is smooth and may be given a smooth compactification by adding a finite number of points called cusps. These points are in $1: 1$ correspondence with elements of $G \backslash \mathbf{P}^{1}(k),\left(\right.$ e.g. $G L_{2}(A)$ has only one cusp etc.).

(2) $X_{G}$ can be given a natural structure as an affine curve over some finite abelian extension of $\mathbf{F}_{r}(T)$.

We also point out that in the rank-one case, these ideas may be used to describe interesting "cyclotomic" abelian extensions of $k$. For the general construction see [1]; for the simpler case of $\mathbf{F}_{r}[T]$, see [3] and also D. Hayes, Trans. Amer. Math. Soc. 189 (1974), 77-91.

\section{Modular forms and $q$-expansions.}

(a) The classical case. Recall the classical definition of a weakly modular form, $f$, of weight $k$ for $\Gamma((n))$. This is a holomorphic function $f$ on $H$ such that if $g=\left(\begin{array}{ll}a & c \\ b & d\end{array}\right) \in \Gamma((n))$, then

$$
f\left(\frac{a z+b}{c z+d}\right)=(c z+d)^{k} f(z) .
$$

Note that because of the action of the units in $\mathbf{Z}$, if $f$ is nontrivial and $n=1$, then $k$ is even.

In particular, $f$ is invariant under the map $z \mapsto z+n$. Thus, $f$ has a Fourier expansion

$$
\sum_{j \in \mathbf{Z}} c_{j}\left(e^{(2 \pi i z / n)}\right)^{j}
$$

More generally, if $b$ is a cusp, we can find $g_{b} \in \mathrm{SL}_{2}(\mathbf{Z})$ so that $g_{b}(b)=\infty$. Let $g_{b}^{-1}=\left(\begin{array}{ll}a & c \\ b & d\end{array}\right)$. Then,

$$
f\left(\frac{a z+b}{c z+d}\right)(c z+d)^{-k}
$$

is also invariant under $z \mapsto z+n$. Thus it too has an expansion in $e^{(2 \pi i z / n)}$, called the expansion at $b$. When there will be no confusion, we denote $e^{(2 \pi i z / n)}$ by $q$.

We now say $f$ is modular iff all the expansions have only finite poles. We say $f$ is holomorphic iff all the expansions have no negative terms. Finally, we say $f$ is a cusp form iff $f$ is holomorphic with value 0 at each cusp.

The theory of such functions is very successful and a great deal is known about them. The next theorem summarizes a small part of these results (see [12]).

THEOREM 4.1. (a) The vector spaces of holomorphic forms of a given weight and level are finite dimensional.

(b) There is a basis of such spaces given by forms whose expansion coefficients are algebraic, (in fact cyclotomic). 
The proof of (a) lies in showing these forms are holomorphic global sections of a line bundle on the compactified modular curves. One then appeals to a G.A.G.A. principle to see that these sections are then given by algebraic maps. Finally then, one knows that spaces of global sections of line bundles are finite-dimensional. The dimension can of ten be computed by the Riemann-Roch theorem.

Recall that every modular curve has a definition over some finite cyclotomic extension of $Q$. The proof of (b) lies with showing that the line bundle also has a definition over the same field. Now, one has an algebraic interpretation of $q$-expansions, via the so-called Tate elliptic curves. We will not give the details except to say that its purpose is to completely reflect the algebraic properties of modular forms. Further, our line bundle certainly has a basis of global sections defined over the cyclotomic field. So, finally, we see the existence of the required forms.

(b) The Laurent-series case. In the case of $\mathcal{H}$ and $k_{\infty}$, we use the same definitions. Let $I$ be a nonzero ideal of $A$. Then, a weakly modular form, of weight $k$, level $I$ is a rigid function $f: \mathcal{H} \rightarrow \mathbf{A}^{1}$, defined over some finite extension of $k_{\infty}$, so that if $\left(\begin{array}{ll}a & c \\ b & d\end{array}\right) \in \Gamma(I)$ then

$$
f\left(\frac{a z+b}{c z+d}\right)=(c z+d)^{k} f(z) \text {. }
$$

Notice that, as with $\mathrm{SL}_{2}(Z)$, for $\mathrm{GL}_{2}(A)$ the weight must be divisible by $(r-1)$ in order for there to exist a nontrivial form.

In particular, for $\mathrm{GL}_{2}(A)$, we see $f$ is invariant under automorphisms of the form $z \rightarrow \zeta z+\alpha ; \zeta \in \mathbf{F}_{r}^{*}, \alpha \in \mathbf{F}_{r}[T]$. In the level $I, I \neq A$, case, it is invariant under translations $z \rightarrow z+\alpha ; \alpha \in I$.

For convenience we will work first in the level $I, I \neq A$, case. Here, we can associate expansions to $f$ in the following manner: We view $I$ as a rank one lattice and we form the function

$$
e_{\infty}(z)=e_{I}(z)=z \prod_{\substack{\alpha \in I \\ 0 \neq \alpha}}(1-z / \alpha) .
$$

Since $e_{\infty}$ is additive, it is invariant under translations

$$
z \rightarrow z+\alpha ; \quad \alpha \in I .
$$

We let $q_{\infty}(z)=e_{\infty}(z)^{-1}$. Recall $e_{\infty}^{\prime}(z)$ is identically 1 . Thus by logarithmic differentation,

$$
q_{\infty}(z)=\frac{e_{\infty}^{\prime}(z)}{e_{\infty}(z)}=\sum_{\alpha \in I}(z+\alpha)^{-1} .
$$

Therefore, as $d(z) \rightarrow \infty, q_{\infty}(z) \rightarrow 0$ uniformly.

Now let $L$ be a finite extension of $k_{\infty}$.

THEOREM 4.2. Let $g(z)$ be a rigid analytic function on $\mathcal{H}$ defined over $L$ and invariant under the maps $z \mapsto z+\alpha ; \alpha \in I$. Then $g$ has an expansion

$$
\sum_{n \in \mathbf{Z}} a_{n} q_{\infty}^{n}(z) ; \quad\left\{a_{n}^{\prime}\right\} \subseteq L
$$


Further, this expansion converges in a nontrivial disc with the origin removed.

For a proof, see [2].

Thus, we deduce $q$-expansions at $\infty$ for our modular forms. We handle the cusps as we did for $\mathrm{SL}_{2}(Z)$, making sure to choose $g_{b} \in \mathrm{SL}_{2}(A)$ so as to avoid any complications.

For $\mathrm{GL}_{2}(A)$, there is a small modification. We set

$$
e_{\infty}(z)=\prod_{\substack{\alpha \in A \\ 0 \neq \alpha}}(1-z / \alpha), \quad g_{\infty}(z)^{-1} \text { and } q_{\infty}=q_{\infty}^{(r-1)} .
$$

The point is that for $\zeta \in \mathbf{F}_{r}^{*}, g_{\infty}(\zeta z)=\zeta^{-1} g_{\infty}(z)$. Thus the $(r-1)$ st power is necessary in order to have a function invariant under

$$
z \rightarrow \zeta z+\alpha ; \quad \zeta \in \mathbf{F}_{r}^{*}, \alpha \in A .
$$

We then say a weakly modular form is modular iff at every cusp the expansion is finite tailed, etc. Further, we can show the existence of a line bundle on the compactified curves so that holomorphic forms are sections of this bundle. We then can argue as before, using a G.A.G.A. principle to conclude the finite dimensionality of the vector spaces of forms.

However, to get the maximum amount of rationality information we have to alter our definitions slightly. For the clue on how to proceed we return briefly to the classical case. Recall that the exponential function has rational Taylor coefficients at the origin. However, it has period $2 \pi i$. To arrive at the "correct" uniformizer $e^{(2 \pi i z)}$, we composed $e^{(z)}$ with the map $z \rightarrow 2 \pi i z$. Now let $I=A$ and consider the function $e_{L}(z)$ of Example 3.4. Recall that it has rational (i.e. $\in \mathbf{F}_{r}(T)$ ) Taylor coefficients at the origin. Recall further that $L=\bar{\lambda} \cdot A$. Therefore, we define $e_{\infty}(\bar{\lambda}, z)$ to be $e_{L}(\bar{\lambda} z), g_{\infty}(\bar{\lambda} ; z)$ to be $e_{\infty}(\bar{\lambda} ; z)^{-1}$ and $q_{\infty}(\bar{\lambda} ; z)$ to be $g_{\infty}(\bar{\lambda} ; z)^{(r-1)}$. (There are appropriate modifications in the case of level $I \neq A$.) The above process is called normalization.

The effect of changing $e_{\infty}(z)$ to $e_{\infty}(\bar{\lambda} ; z)$ merely multiplies $e_{\infty}(z)$ by $\bar{\lambda}$; i.e. $e_{\infty}(\bar{\lambda} ; z)=\bar{\lambda} e_{\infty}(z)$, etc. Further, if $f=\sum a_{n} q_{\infty}^{n}$ then $f=\sum a_{n}(\bar{\lambda})^{n(r-1)} q_{\infty}(\bar{\lambda} ; z)^{n}$. However, with this modification one can now proceed as classically to deduce the following theorem (see [2]).

THEOREM 4.3. (a) The vector spaces of holomorphic forms of a given weight and level are finite dimensional.

(b) There is a basis of such spaces given by forms whose normalized expansion coefficients are algebraic.

Again we may just as easily work with the modules $\left(C_{\zeta}\right)$. One sees easily that what is intrinsic is the fractional ideal (or finite divisor) generated by the coefficients. Thus, the coefficients may be thought of as geometric objects. The full impact of this fact is not yet clear.

5. Cusp forms and double-cusp forms. Let $n \in \mathbf{N}$ and $\alpha=\left(\begin{array}{ll}a & c \\ b & d\end{array}\right) \in \Gamma((n))$. Thus, $\alpha(z)=(a z+b) /(c z+d)$. Since $\operatorname{det}(\alpha)=1$, we see

$$
d(\alpha(z))=d z /(c z+d)^{2} .
$$

Therefore, if $f$ is a form of weight 2 , level $(n), f d z$ is an invariant differential; i.e. is a differential form on $X_{\Gamma((n))}$. Now let $b$ be a cusp and $q$ the uniformizer 
there. One has $d q=c q d z$ for some nonzero constant $c$. Therefore, $d z=$ $d q / c q$, and if $f$ is a cusp form then $f d z$ is a holomorphic 1-form on the compactification of $X_{\Gamma((n))}$. The converse is easy to see. So we have

THEOREM 5.1. The dimension of the space of cusp forms of weight 2 is $g$, where $g$ is the genus of $\overline{X_{\Gamma((n))}}$.

In the function field case, we need to have level $I \neq A$ structure in order to have determinant equal to 1 . This amounts to the easy observation that $\mathbf{F}_{r}^{*}$ injects into $\mathbf{F}_{r}[T] / I$. Under this assumption, if $f$ is a form of weight 2 , level $I$, then $f d z$ is an invariant differential. Now let $b$ be a cusp and $q_{b}$ the uniformizer at $b$. Since $e_{b}^{\prime}$ is identically 1 and $q_{b}=e_{b}^{-1}$, we find

$$
d q_{b}=-1 q_{b}^{2} d z
$$

Thus, if $f$ is a holomorphic form with two zeroes at each cusp, $f d z$ is a holomorphic differential on the compactification of $X_{\Gamma((I))}$.

Definition 5.2. A holomorphic form of level $I$, is a double-cusp form iff it has a double zero at each cusp.

Thus,

THEOREM 5.3. The dimension of the space of double-cusp forms of weight 2, level $I$, is the genus of $X_{\Gamma(I)}$.

The concept of double-cusp form seems to have little utility in the case of the full group $\mathrm{GL}_{2}(A)$. However, any cusp form for the full group becomes double-cuspidal at each level $I \neq A$.

6. Eisenstein series and additive harmonic analysis.

(a) Classical Eisenstein series. Our purpose here is to discuss Eisenstein series and their expansions. In the function field case, this will lead into a discussion of "additive harmonic analysis".

The simplest examples of modular forms for $\mathrm{SL}_{2}(Z)$ or principal congruence subgroups, are the Eisenstein series. They are defined as follows.

DeFinition 6.1. (a) For $\mathrm{SL}_{2}(\mathrm{Z})$ let $k \geqslant 2$. We set

$$
E_{2 k}(z)=\sum_{(c, d) \in \mathbf{Z} \oplus \mathbf{Z}}^{\prime}(c z+d)^{-2 k}
$$

where $\Sigma^{\prime}$ means summation over nonzero elements.

(b) Let $n \geqslant 2$ and let $(0,0) \neq\left(a_{0}, a_{1}\right) \in \mathbf{Z} /((n)) \oplus \mathbf{Z} /((n))$. Let $k \geqslant 3$. Then, for level $(n)$, we set

$$
E_{k}\left(z ; a_{0}, a_{1} ; n\right)=\sum_{\substack{c \equiv a_{0}(n) \\ d \equiv a_{1}(n)}}(c z+d)^{-k} .
$$

It is classical that these series are holomorphic on $\boldsymbol{H}$. It is further easy to see they satisfy the correct modular rules. From classical cotangent formulae one computes their $q$-expansions (see [9]). We recall these below. 
THEOREM 6.2. (a)

$$
E_{2 k}(z)=2 \sum_{n \in \mathbf{N}}^{\prime} n^{-2 k}+\frac{2(2 \pi i)^{2 k}}{(2 k-1) !} \sum_{n=1}^{\infty} \sigma_{2 k-1}(n) q^{n},
$$

where $\sigma_{v}(n)=\Sigma_{d \mid n ; d>0} d^{v}$.

(b) Let $q=e^{(2 \pi i z / n)}$. Then $E_{k}\left(z ; a_{0}, a_{1} ; n\right)=\sum_{\lambda=0}^{\infty} a_{\lambda} q^{\lambda}$, where

$$
a_{0}=\left\{\begin{array}{l}
0 \text { if } a_{0} \not \equiv O(n) \\
\sum_{\alpha \equiv a_{0}(n)} \alpha^{-k} \text { if } a_{0} \equiv O(n)
\end{array}\right.
$$

and, for $\lambda \geqslant 1$,

$$
a_{\lambda}=\frac{(-2 \pi i)^{k}}{n^{k}(k-1) !} \sum_{\substack{m v=\lambda \\ m \equiv a_{0}(n)}}(\operatorname{sgn} v) v^{k-1} e^{\left(2 \pi i a_{1} v / n\right)} .
$$

Note that our computation in (b) is only at $\infty$. But, passing from $\infty$ to another cusp permutes the Eisenstein series. Therefore, our computation is sufficient. Thus, the Eisenstein series are holomorphic forms. Further, it is apparent that upon dividing by powers of $\pi$ we obtain rational coefficients in (a) and algebraic coefficients in (b).

(b) Characteristic-p Eisenstein series. Now we pass to the characteristic- $p$ analogue. We shall see that the theory here seems to behave very differently. In fact, not much specific information about the $q$-expansions is known. We will content ourselves with sketching the computation and presenting a few properties.

Definition 6.3. (a) For $\mathrm{GL}_{2}(A)$, we set

$$
E_{(r-1) k}(z)=\sum_{(c, d) \in A \oplus A}^{\prime}(c z+d)^{-(r-1) k} .
$$

(b) Let $I \neq A$ be an ideal and $(0,0) \neq\left(a_{0}, a_{1}\right) \in A / I \oplus A / I$. Then, for level $I$, we set

$$
E_{k}\left(z ; a_{0}, a_{1} ; I\right)=\sum_{\substack{c \equiv a_{0}(I) \\ d \equiv a_{1}(I)}}(c z+d)^{-k}
$$

The function $g(z)=c z+d$ is rigid analytic and never zero on $\mathcal{H}$, so $(c z+d)^{-1}$ is rigid analytic. Further, $(c z+d)=c(z+d / c)$. So, $|c z+d|=$ $|c||z+d / c|$. As $d / c \in \mathbf{F}_{r}(T),|z+d / c| \geqslant d(z)$. Thus $|c z+d| \geqslant|c| d(z) \rightarrow$ $\infty$ as $|c| \rightarrow \infty$. By the nonarchimedean property of || , we see our series converge uniformly on $U_{i}$, as defined in Proposition 2.4.b, and so define weakly modular functions on $\mathcal{H}$.

(c) Additive harmonic analysis. In order to actually compute the $q$-expansions, we need to discuss a harmonic analysis based on addition. Our main computational tool will be the classical formulae of Newton, which we now recall. (The reader may verify them as an exercise.) 
6.4. NewTON'S FORMULAE. Let $f(x)=x^{n}+a_{1} x^{n-1}+\cdots+a_{n}$ be a polynomial and let $\alpha_{1}, \ldots, \alpha_{n}$ be its roots. Further, let $S_{k}=\alpha_{1}^{k}+\cdots+\alpha_{n}^{k}$. Then

(a) $S_{k}+a_{1} S_{k-1}+\cdots+a_{k-1} S_{1}+k a_{k}=0, k=1, \ldots, n$

(b) $S_{k}+a_{1} S_{k-1}+\cdots+a_{n} S_{k-n}=0, k>n$.

Now let $M$ be a finite $\mathbf{F}_{r}$ vector subspace of $\bar{k}_{\infty},|M|$ the number of elements in it, $e_{M}(z)=z \prod_{0 \neq \alpha \in M}(1-z / \alpha), Q_{h}(z)=\sum_{\alpha \in M}(z+\alpha)^{-h}$ and $q_{M}(z)=e_{M}(z)^{-1}$.

Proposition 6.5. There is a monic polynomial, of degree $h, P_{h}(x)$, such that $P_{h}\left(e_{M}\right)=Q_{h}$. Further, $P_{h}(0)=0$.

Construction Proof. Let $w$ be another transcendental element. Then, since $e_{M}(z)$ is an additive function, we see

$$
e_{M}(w-z)=e_{M}(w)-e_{M}(z)=(w-z) \prod_{0 \neq \alpha \in M}\left(1-\frac{(w-z)}{\alpha}\right) .
$$

So, as a polynomial in $w, e_{M}(w)-e_{M}(z)$ has roots $z+\alpha, \alpha \in M$.

Now set $\log _{r}|M|=e$ and $e_{M}(z)=\sum_{i=0}^{e} a_{i} z^{r^{i}}$. Then, $1 /(z+\alpha)$ is a reciprocal root of

$$
e_{M}(w)-e_{M}(z)=\sum a_{i} w^{r^{i}}-e_{M}(z) .
$$

Therefore, by simple algebra, $1 /(z+\alpha)$ is a root of

$$
w^{r^{h}}+\sum_{i=0}^{e}-a_{i} q_{M} w^{\left(r^{h}-r^{i}\right)}
$$

Newton's formulae now finishes the proof.

As a corollary of the proof, we see that the coefficients of $P_{h}$ are polynomials in the coefficients of $e_{M}$. Further, we can see by construction that $P_{h}(x)=x^{h}$, for $1 \leqslant h \leqslant r$. In particular, we obtain a different proof of the fact that $e_{M}(z)^{-1}=\sum_{\alpha \in M}(z+\alpha)^{-1}$.

Now let $N=\mathbf{F}_{r}[T] \cdot x$ for some $x \in \bar{k}_{\sigma}^{*}$ and let $e_{N}(z)$ be the associated function. We set, as rigid meromorphic functions, $Q_{h}(z)^{\prime}=\Sigma_{\alpha \in N}(z+\alpha)^{-h}$ and $q_{N}(z)=e_{N}(z)^{-1}$.

Proposition 6.6. There exists a monic polynomial, $P_{h}$, so that $P_{h}\left(q_{N}(z)\right)=$ $Q_{h}(z)$.

Construction proof. Write $N=\cup N_{i}$ with a finite $\mathbf{F}_{r}$ vector space. Thus all our functions are limits of their $N_{i}$-analogs. Now notice that once $\left|N_{i}\right|>h$, the formula given above for the coefficients of $P_{h}$ in terms of the Taylor series of $e_{N_{i}}(z)$ is invariant of $N_{i}$. Further, passing to the limit causes no problem. Thus, we can use this formula on $e_{N}$ to find $P_{h}$.

Corollary of Proof 6.7. If $e_{N}$ has coefficients in a field $K$, then so does $P_{h}$.

(d) q-expansions in finite characteristic. We can now compute the expansions of the Eisenstein series. We shall first give a very general formula, then work out an example in detail. Thus, let $I$ be a nonzero ideal, which may be $A$ itself. Let $\left(a_{0}, a_{1}\right) \in A \oplus A$ and, for now, put

$$
E(z)=\sum_{(c, d) \equiv\left(a_{0}, a_{1}\right)(\bmod I)}^{\prime}(c z+d)^{-h} .
$$


Let $g_{\infty}(z)=\Sigma_{\alpha \in I}(z+\alpha)^{-1}=e_{\infty}(z)^{-1}$. Finally, let $P_{h}$ be chosen as in Proposition 6.6 for $N=I$. Now if $a_{0} \equiv O(I)$, then $\Sigma_{d \equiv a_{1}(I)} d^{-j}$ is the constant term. Otherwise, the constant term is zero. Next suppose $c \neq 0$; then $\Sigma_{d \equiv a_{1}(I)}(c z+d)^{-h}=c^{-h} \sum_{d \equiv a_{1}(I)}(z+d / c)^{-h}$. We let $\left\{x_{i}^{c}\right\}$ be representatives of $I / c I$. So, by Proposition 6.6 we have,

$$
\begin{aligned}
\sum_{d \equiv a_{1}(I)}(z+d / c)^{-h} & =\sum_{\left\{x_{i}^{c}\right\}} \sum_{\alpha \in I}\left(z+\frac{a_{1}}{c}+\frac{x_{i}^{c}}{c}+\alpha\right)^{-h} \\
& =\sum_{\left\{x_{i}^{c}\right\}} P_{h}\left(g_{\infty}\left(z+\frac{a_{1}}{c}+\frac{x_{i}^{c}}{c}\right)\right)
\end{aligned}
$$

Suppose $P_{h}(x)=\sum_{j=1}^{h} b_{j} x^{j}$. Then the last sum is

$$
\sum_{\left\{x_{i}^{c}\right\}} \sum_{j=1}^{h} b_{j} g_{\infty}\left(z+a_{1} / c+x_{i}^{c} / c\right)^{j} .
$$

But,

$$
\begin{aligned}
g_{\infty}\left(z+a_{1} / c+x_{i}^{c} / c\right) & =e_{\infty}\left(z+a_{1} / c+x_{i}^{c} / c\right)^{-1} \\
& =\left(e_{\infty}(z)+e_{\infty}\left(a_{1} / c+x_{i}^{c} / c\right)\right)^{-1} \\
& =g_{\infty}(z)\left(1+g_{\infty}(z) e_{\infty}(z) e_{\infty}\left(a_{1} / c+x_{i}^{c} / c\right)\right)^{-1} .
\end{aligned}
$$

Thus, the geometric series allows us to expand $g_{\infty}\left(z+a_{1} / c+x_{i}^{c} / c\right)$ as a power-series in $g_{\infty}$, and thus to expand its powers. Summing over $c$ gives us the expansion of $E(z)$ in terms of $g_{\infty}$. Note that in the case $I=A$, the uniformizer at infinity is $q_{\infty}=g_{\infty}^{(r-1)}$. However, since $E(\zeta z)=E(z), g_{\infty}(\zeta z)=$ $\zeta^{-1} g_{\infty}(z)$, for $\zeta \in \mathbf{F}_{r}^{*}$, we see that the above expansion ends up in terms of $q_{\infty}$. In the case $I \neq A, g_{\infty}=q_{\infty}$. Finally, reasoning as for congruence subgroups of $\mathrm{SL}_{2}(Z)$, we see that this computation is general. We have shown

THEOREM 6.8. The Eisenstein series are holomorphic at the cusps.

Example 6.9. Let $I=A$ and $j=(r-1)$. Our function then is the first nontrivial Eisenstein series $E_{j}$. We have mentioned that $P_{j}(x)=x^{(r-1)}$ and so

$$
E_{j}(z)=\sum_{c \neq 0} c^{-j}+\sum_{c=0} q_{\infty}(c z)
$$

Now it is often possible to go the general computation one step further; to directly, give the expansion in terms of $q_{\infty}$. We shall do this here. It is based on the following lemma that the reader may easily check.

LEMMA 6.10. Let $b=x^{-(r-1)}$. Then

$$
\sum_{\zeta \in \mathbf{F}_{r}^{*}}(x+\zeta)^{-(r-1)}=\frac{b-1}{b^{r}-1}-1 .
$$

$\mathbf{F}_{r}^{*}$ acts on $I / c I$ and we let $\left\{\bar{x}_{i}^{c}\right\}$ be a set of representatives of $\mathbf{F}_{r}^{*} \backslash(I / c I)$; i.e. of the associated quotient classes. Since $e_{\infty}$ is $\mathbf{F}_{r}$-linear, we have

$$
\sum_{\left\{x_{i}^{c}\right\}}\left(e_{\infty}\left(z+x_{i}^{c} / c\right)\right)^{-(r-1)}=\sum_{\left\{\bar{x}_{i}^{c}\right\}} \sum_{\zeta \in \mathbb{F}_{r}^{*}}\left(e_{\infty}(z)+\zeta e_{\infty}\left(\bar{x}_{i}^{c} / c\right)\right)^{-(r-1)} .
$$


We now put $x=e_{\infty}(z) / e_{\infty}\left(\bar{x}_{i}^{c} / c\right)$, use the lemma and then the geometric series to conclude,

$$
\begin{aligned}
q_{\infty}(c z)=c^{-(r-1)} q_{\infty}+c^{-(r-1)} & \sum_{\left\{\begin{array}{c}
\left\{\bar{x}_{i}^{c}\right\} \\
\bar{x}_{i}^{c} \neq 0
\end{array}\right\}}\left(-q_{\infty}+e_{\infty}\left(\bar{x}_{i}^{c} / c\right)^{(r-1)^{2}} q_{\infty}^{r}\right. \\
& \left.-e_{\infty}\left(\bar{x}_{i}^{c} / c\right)^{r(r-1)} q_{\infty}^{(r+1)}+\cdots\right) .
\end{aligned}
$$

Notice that if $c$ is not a unit, then the number of nonzero elements in $\left\{\bar{x}_{i}^{c}\right\}$ is $\equiv 1(r)$; so $q_{\infty}(c z)$ has at least two zeroes as a function of $q_{\infty}$. Therefore,

$$
\begin{aligned}
E_{(r-1)}(z)= & \sum_{c \neq 0} c^{-(r-1)}+\left(\sum_{c \in \mathbf{F}_{r}^{*}} c^{-(r-1)}\right) q_{\infty} \\
& +\sum_{c \notin \mathbf{F}_{r}^{*}} c^{-(r-1)} \sum_{\left\{\bar{x}_{i}^{c}\right\}} e_{\infty}\left(\bar{x}_{i}^{c} / c\right)^{(r-1)^{2}} q_{\infty}^{r} \cdots .
\end{aligned}
$$

But, if $c \in \mathbf{F}_{r}^{*}, c^{(r-1)}=1$. Thus, the coefficient of $q_{\infty}$ is $(r-1)=-1(r)$.

What about the higher coefficients? The key is to notice that $e_{\infty}\left(\bar{x}_{i}^{c} / c\right)$ is a point of order $c$ on the elliptic module associated to $A$ (viewed as a lattice). Further, all the powers that occur are divisible by $(r-1) \cdot A s(r-1) \equiv-1$ ( $r$ ) we may compute the sums above by summing over all the elements $\left\{x_{i}^{c}\right\}$ and then multiply by -1 . But now we are computing power sums of roots of polynomials and so, again, we may use Newton's rules.

Our first corollary is very important: Let $\phi$ be the elliptic module associated to $A$ and let $c \in A$. We know $\phi(c)(z)=c z+\sum_{i=1}^{D(c)} a_{i} z^{r^{i}}$. Thus, the gap between the highest nonzero term and next highest is $r^{D(c)}-r^{D(c)-1}$; which tends to infinity with $D(c)$. But Newton tells us that once $i \leqslant r^{D(c)}-$ $r^{D(c)-1}$, the sums of the $i$ th power of the roots of $\phi(c)(z)=0$ is 0 . Thus, in any coefficient, only finitely many c's may have a nonzero contribution.

Finally, when we normalize our uniformizer and divide by $(\bar{\lambda})^{(r-1)}$, we see that the coefficients of $q_{\infty}(\bar{\lambda} ; z)^{n}, n \geqslant 1$, may be computed by using Carlitz's module $C$. In other words we use Newton to find the same power sums of $C(a)(z)=0, a \in A$, as we used for $\phi$. As a corollary, these normalized coefficients are rational; i.e. in $\mathbf{F}_{r}(T)$.

It remains to discuss the constant term. Upon dividing by $(\bar{\lambda})^{r-1}$, we are reduced to computing

$$
\sum_{0 \neq \alpha \in L} \alpha^{-(r-1)}
$$

where $L$ is the lattice associated to $C$. Now, we know that

$$
\frac{1}{e_{L}(z)}=\sum_{\alpha \in L}(z+\alpha)^{-1}
$$

Therefore, we can use the geometric series to see that the Laurent coefficients, at the origin, of $1 / e_{L}(z)$ are sums of the form $\pm \Sigma_{\alpha \in L} \alpha^{-k}$. But, we can compute these directly from $e_{L}$ by synthetic division. This gives us the last tool needed to find the coefficients. (For more on the constant terms, i.e. "zeta-values", see [4].) For an analytic theory of zeta functions developed along these lines see $v$-adic zeta functions, L-series, and measures for function 
fields, Invent. Math. 55 (1979), 107-120. In particular, we see that the normalized coefficients are rational multiples of $(\bar{\lambda})^{(r-1)}$.

The above procedure works in general for all Eisenstein series for $\mathrm{GL}_{2}$. Thus we have

THEOREM 6.11. The normalized coefficients of $E_{i(r-1)}(z)$ are rational multiples of $(\bar{\lambda})^{i(r-1)}$.

For instance, set $r=3$. We find by using the above procedure,

$$
\begin{aligned}
(\bar{\lambda})^{-2} E_{2}(z)= & \frac{-1}{\left(T^{9}-T\right)\left(T^{3}-T\right)}-q_{\infty}(\bar{\lambda}, z)+O \cdot q_{\infty}(\bar{\lambda}, z)^{3} \\
& +O \cdot q_{\infty}(\bar{\lambda}, z)^{4}+O \cdot q_{\infty}(\bar{\lambda}, z)^{6}-q_{\infty}(\bar{\lambda}, z)^{7}+\cdots .
\end{aligned}
$$

Very mysterious!

What about the case of the principal congruence subgroups? The theory is essentially the same for the nonconstant terms. The constant term is handled by the following lemma.

LEMMA 6.12. Let $J$ be a rank one lattice and let $\beta \in k \otimes J-J$. Then

(a) $1 / e_{J}(\beta)=\sum_{\alpha \in J} 1 /(\beta+\alpha)$.

(b) In general, the sums $\Sigma_{\alpha \in J}(\beta+\alpha)^{-i}$ occur as Taylor coefficients at the origin, of the function in $z\left(e_{J}(z)+\epsilon_{N}(\beta)\right)^{-1}$.

Proof. (a) follows directly from the formula

$$
e_{J}(z)^{-1}=\sum_{\alpha \in J}(z+\alpha)^{-1},
$$

upon setting $z=\beta$.

To see (b) we notice that for $\beta \in k \otimes J-J$,

$$
\left(e_{J}(z)+e_{J}(\beta)\right)^{-1}=e_{J}(z+\beta)^{-1}=\sum_{\alpha \in J}(z+\beta+\alpha)^{-1} .
$$

Now, we again use the geometric series to express both sides about the origin and compare coefficients.

With the lemma, the general computation is finished and we find

THEOREM 6.13. The normalized coefficients of the Eisenstein series of weight $i$, level I, are ith powers of a fixed constant times an algebraic element.

In comparison with the classical case, the expansion coefficients are very puzzling. For the full group, the denominators of the constant terms are computed in [4], and, in [3], this result is extended to all the coefficients. Still, nothing very specific is known. For instance, are the nonconstant terms actually in $\mathbf{F}_{r}[T]$ ? (This would imply a terrific amount of concellation.) When are they zero? What primes divide them? etc.

7. Hecke operators. (a) Hecke operators for congruence subgroups of $S L_{2}(Z)$. One of the most beautiful aspects of the classical theory of modular forms is the theory of the Hecke operators. These operators act on spaces of modular forms and yield a terrific amount of arithmetic information. We will now summarize this. 
For $\mathrm{SL}_{2}(\mathrm{Z})$ the definition is as follows. Let $f(z)$ be a form of weight $2 k$ and let $n \in \mathbf{N}$. Then,

$$
T((n)) f(z)=n^{2 k-1} \sum_{\substack{a>1, a d=n \\ 0<b<d}} d^{-2 k} f\left(\frac{a z+b}{d}\right) .
$$

As, $T((n))$ is defined by summing over all sublattices of index $n$, it is simple to see that $T((n)) f(z)$ is also a form of weight $2 k$ for $\mathrm{SL}_{2}(\mathbf{Z})$. Further, we have

(a) $T((m)) T((n)) f=T((m n)) f$, if $(m, n)=1$,

(b) $T((p)) T\left(\left(p^{n}\right)\right) f=T\left(\left(p^{n+1}\right)\right) f+p^{2 k-1} T\left(\left(p^{n-1}\right)\right) f$,

if $p$ is a prime and $n \geqslant 1$.

(Indeed, (a) is simple. (b) is proved by taking into account multiplicities; see [11].) Therefore, we see these operators commute. Now, let $f(z)=$ $\Sigma_{m \in \mathbf{Z}} c_{m} q^{m}, q=e^{(2 \pi i z)}$. Then, by using standard formulae on sums of powers of roots of unity, one computes that

$$
T((n)) f(z)=\sum_{m \in \mathbf{Z}} \gamma_{m} q^{m}
$$

with

$$
\gamma_{m}=\sum_{\substack{a \mid(n, m) \\ a>1}} a^{2 k-1} C_{m n / a^{2}}
$$

(See [11, pp. 98-102].)

From the calculation we derive the important corollaries. (1) $T((n))$ takes holomorphic forms to holomorphic forms and cusp forms to cusp forms. (2) Let $n=p, p$ prime. Then $\gamma_{m}=c_{p m}$ if $m \neq 0(p) ; \gamma_{m}=c_{p m}+p^{2 k-1} c_{m / p}$ if $m \equiv 0(p)$.

Suppose, finally, that $f$ is a nonzero eigenfunction for all $T((n))$; i.e. $T((n)) f=\lambda_{n} f$. Then we find $c_{1} \neq 0$ and, if we normalize so that $c_{1}=1$, then $c_{n}=\lambda_{n}$ for all $n$. Thus, we find an important dictionary between eigenvalues and $q$-expansion coefficients.

The simplest nontrivial examples of such eigenfunctions are the Eisenstein series. One computes, (see [11, p. 104]), that

$$
T((n)) E_{2 k}=\sigma_{2 k-1}(n) E_{2 k} .
$$

On the space of cusp forms, there exists an inner product, called the Peterson product, for which the Hecke operators are hermitian. One thus deduces the existence of an orthogonal basis of cusp forms consisting of eigenfunctions for all the Hecke operators.

In the case of level (m), $m \in \mathbf{N}$, one can define Hecke operators for any number prime to $\mathrm{m}$, in a very similar manner. With this many of the above results go thru; e.g. the space generated by Eisenstein series of a given weight is stable under the Hecke operators. The space of cusp forms is also stable, etc. We refer the reader to [9].

(b) Hecke operators in the Laurent-series case. Before returning to the function field case, we give a useful convention: lower English letters will now denote monic elements of $A$, if they denote an element of $A$.

Therefore, let $f(z)$ be a weakly modular form of weight $j=(r-1) j_{0}$ for the full group $\mathrm{GL}_{2}(A)$. Let $i \in A$. Our definition here is similar to the classical 
one; we differ only in using "overlattices" as opposed to "sublattices". So, we set

$$
T((i)) f=\sum_{\substack{a, a d=i \\ D(B)<D(d)}} a^{j} f\left(\frac{a z+B}{d}\right) .
$$

It is immediate that $T((i)) f$ is weakly modular on $\mathcal{H}$.

One of the interesting facets of the theory over the formal Laurent-series fields, is that $T((i)) T((h)) f=T((i h)) f$ for all $i, h \in A$. The reason for this is quite amusing; we perform the standard calculation and we see that the multiplicities are either 1 or 0 modulo (r). As $r=0$ in $A$, the result follows.

In the case at hand, one cannot as yet present a formula on the effect on $q$-expansions as beautifully simple as the one for the $\mathrm{SL}_{2}(\mathbf{Z})$ case. Still we can give a procedure for the calculation. Thus, let $f=\Sigma_{n \geqslant 0} c_{n} q_{\infty}^{n} ; q_{\infty}=g_{\infty}^{(r-1)}$, $g_{\infty}=e_{\infty}^{-1}$.

LEMMA 7.1. The sum $\Sigma_{D(B)<D(d)} q_{\infty}((a z+B) / d)$ is a polynomial in $q_{\infty}(a z)$.

Computation-Proof. Let $\phi$ be the elliptic module associated to $e_{\infty}$; i.e. $e_{\infty}(a z)=\phi(a)\left(e_{\infty}(z)\right)$ for $a \in A$. Now, the elements $g_{\infty}((a z+B) / d)$ are reciprocal roots of the polynomial in $x, \phi(d)(x)-e_{\infty}(a z)=0$. Suppose, $\phi(d)=\sum_{i=0}^{D(d)} a_{i} x^{r^{i}}$. Then, by simple algebra, the reciprocal roots of $\sum a_{i} x^{r^{i}}-$ $e_{\infty}(a z)$ satisfy

$$
x^{r^{D(d)}}-\sum a_{i} g_{\infty}(a z) x^{\left(r^{D(d)}-r^{\prime}\right)} .
$$

Again, by Newton, we can compute power sums of $g_{\infty}((a z+B) / d)$ in terms of $g_{\infty}(a z)$ and so we can compute power sums of $q_{\infty}((a z+B) / d)$. Reasoning as we did for the Eisenstein series, we see the power sums of $q_{\infty}((a z+B) / d)$ end up as expressions in $q_{\infty}(a z)$. This gives the result.

Thus, we are reduced to finding the expansions of $q_{\infty}(a z)^{n}, n \geqslant 1, a \in A$. More generally we have:

LemmA 7.2. Let $0 \neq a \in A$ and let $\alpha_{1}, \ldots, \alpha_{n}$ be representatives of $A /(a)$. Then

$$
g_{\infty}(a z)=\frac{1}{a} \sum_{\alpha_{i}}\left(g_{\infty}(z)-e_{\infty}\left(\alpha_{i} / a\right) g_{\infty}^{2}(z)+\cdots\right)
$$

Proof. We have seen $g_{\infty}(z)=\Sigma(z+\alpha)^{-1}$. So

$$
\begin{aligned}
g_{\infty}(a z) & =\frac{1}{a} \sum(z+\alpha / a)^{-1}=\frac{1}{a} \sum_{\left\{\alpha_{i}\right\}} \sum_{\alpha}\left(z+\alpha_{i} / a+\alpha\right)^{-1} \\
& =\frac{1}{a} \sum_{\left\{\alpha_{i}\right\}} e_{\infty}\left(z+\alpha_{i} / a\right)^{-1}=\frac{1}{a} \sum_{\left\{\alpha_{i}\right\}}\left(e_{\infty}(z)+e_{\infty}\left(\alpha_{i} / a\right)\right)^{-1} \\
& =\frac{1}{a} \sum_{\left\{\alpha_{i}\right\}} g_{\infty}(z)\left(1+e_{\infty}\left(\alpha_{i} / a\right) g_{\infty}(z)\right)^{-1} .
\end{aligned}
$$

The geometric series then finishes the proof.

Consequently, we can compute $g_{\infty}(a z)^{n}$ in terms of $g_{\infty}$; the same result is 
true for $q_{\infty}$. If we normalize, we just do the whole computation with Carlitz's module $\mathrm{C}$.

As for $\mathrm{SL}_{2}(\mathbf{Z})$, the Eisenstein series are again eigenforms for all the Hecke operators. Further, one sees that for $j=(r-1) j_{0}, T((i)) E(z)=i^{j} E(z)$, for all $i \in A$. (Note that $i^{j}$ depends only on (i).) The reason is that we do the standard computation for $\mathrm{SL}_{2}(\mathbf{Z})$ and, as with the Hecke operators, we see some multiplicities are divisible by $r$.

The case of level $I$ is handled in the same fashion as for principal congruence subgroups of $\mathrm{SL}_{2}(Z)$; see [2]. The effect on expansions is calculated in the same manner as above. One then can see, as for congruence subgroups of $\mathrm{SL}_{2}(Z)$, that the space generated by Eisenstein series is stable under the Hecke operators. The space of cusp forms is also stable. Further, by checking Newton closely, we see that the space of double-cusp forms is also stable. In view of the classical situation this is very surprising.

It seems unlikely that there are bases for these spaces consisting of eigenvectors (though miracles do sometimes happen!). Still, we are guaranteed some eigenvectors. This follows from the same result on nontrivial finite dimensional representations of commutative algebras.

The action of the Hecke operators, like the $q$-expansions, is very mysterious. For instance, let $\Delta=\left(T^{r^{2}}-T^{r}\right) E_{(r-1)}^{(r+1)}-\left(T^{r^{2}}-T\right) E_{r^{2}-1}$. Then, $\Delta$ is a form of weight $\left(r^{2}-1\right)$ for the full group. One can show that $\Delta$ is nowherezero on $\mathcal{H}$ and is a cusp form. Further, one can show that $\left\{k_{\infty} \cdot \Delta\right\}$ is the space of cusp forms of weight $r^{2}-1$. Thus, $\Delta$ must be an eigenform for the Hecke operators. Consequently, $\Delta$ acts like the classical $\Delta=(60)^{3} E_{2}^{3}-27$. $(140)^{2} E_{3}^{2}$. However, in [3], it is shown that $T((i)) \Delta=i^{(r-1)} \Delta$, for all $i$. As a consequence, $E_{r-1}$ and $\Delta$ have the same eigenvalues for all $T((i))$. This never happens classically. It means that the Hecke operators alone do not distinguish a modular form. Further, it seems to imply, as Serre remarked, that the Hecke operators act like "grossencharacters of type $A_{0}$ ".

Still, given an eigenfunction for all the Hecke operators, there must be many relations among the coefficients. Perhaps, when finally understood, these relations will not be as complicated as they at first appear. One might then see exactly how much information the Hecke operators are giving.

Complements. Although the theory over a Laurent-Series field contains many analogues of classical objects, some analogues are not known to exist. For instance, do these series correspond in some way to Galois representations; or, for that matter, anything involving the Galois groups? Are there analogues of theta functions, and do these analogues give us "sums of squares"-type formulae? Is there a Poisson summation formula, or a Mellin transform? etc. Such questions are very intriguing.

\section{REFERENCES}

1. V. G. Drinfeld, Elliptic modules, Math. Sb., 94 (1974), 594-627. (Russian) (English translation, Math USSR, Sb. 23 (1974), No. 4.)

2. D. Goss, $\pi$-adic Eisenstein series for function fields, Compositio (to appear).

3. __ Modular forms for $\mathbf{F}_{r}[t]$, Crelle's Journal.

4. __ , von Staudt for $\mathbf{F}_{q}[T]$, Duke Math. J. 45 (1978), 885-910. 
5. H. Grauert and L. Gerritzen, Die Azyklizität der affinoiden Überdeckungen, Global Analysis, Univ. of Tokyo Press, 1969, pp. 159-184.

6. R. Kiehl, Der Endlichkeitsatz für eigenliche Abbildungen in der nichtarchimedischen Funktionen Theorie, Invent. Math. 2 (1967), 191-214.

7. Theorem A and Theorem B in der nichtarchimedischen Funcktionen Theorie, Invent. Math. 2 (1967), 256-273.

8. S. Lang, Algebra, Addison-Wesley, Mass., 1965.

9. A. Ogg, Modular forms and Dirichlet series, Benjamin, New York, 1969.

10. A. Robert, Elliptic curves, Lecture Notes in Math. vol. 326, Springer-Verlag, Berlin and New York, 1973, pp. II. 70-II. 85.

11. J.-P. Serre, A course in arithmetic, Springer-Verlag, Berlin and New York, 1973.

12. G. Shimura, Introduction to the arithmetic theory of automorphic functions, Publ. Math. Soc. Japan, $N^{0} 11$, Tokyo-Princeton, 1971.

13. J. Tate, Rigid analytic spaces, Invent. Math. 12 (1971), 257-258.

Department of Mathematics, University of California, Berkeley, California 94720 
